\title{
Sea state bias in altimetry measurements within the theory of similarity for wind-driven seas
}

\author{
Sergei I. Badulin,b,*, Vika G. Grigorieva ${ }^{\mathrm{a}}$, Pavel A. Shabanov ${ }^{\mathrm{a}}$, Vitali D. \\ Sharmar $^{\mathrm{a}}$, Ilya O. Karpov ${ }^{\mathrm{a}}$ \\ ${ }^{a}$ Shirshov Institute of Oceanology, Russian Academy of Sciences, 36, Nakhimovskii pr., \\ 117997 Moscow \\ ${ }^{b}$ Skolkovo Institute of Science and Technology, Bolshoy Boulevard 30, bld. 1, 121205 \\ Moscow, Russia
}

\begin{abstract}
The theory of similarity for wind-driven seas is applied to the physical analysis of the problem of sea state bias (SSB) in altimetry measurements. Dimensionless wave steepness and pseudo-age derived from altimetry measurements are expected to provide physically relevant and accurate enough SSB estimates. Analysis of Jason-1,2,3 and SARAL/AltiKa data within the approach shows the similarity and robustness of SSB distributions re-casted onto space of wave pseudo-age and steepness. This result is considered as a ground for developing a new parametric model of SSB and for analysis of underlying physical effects.
\end{abstract}

Keywords: sea state bias, satellite altimetry, wave steepness, pseudo-age of wind waves, similarity approach

\section{Introduction}

Sea state bias (SSB) in radar altimeter measurements is one of the most complex sources of the ranging errors. Being of the order of a few percents of significant wave height it is the largest remaining error in mean sea level estimates (Gommenginger, Srokosz, Bellingham et al., 2018).

Three main effects are assumed to be responsible for SSB. Electromagnetic bias (EMB) is associated with different reflectivity of wave crests and

\footnotetext{
${ }^{*}$ Corresponding author: Sergei I. Badulin

Email address: badulin.si@ocean.ru (Sergei I. Badulin)
} 
troughs and resulting in a permanent overestimation of satellite-to-surface range measured by altimeter. Skewness bias (SB) is connected with the inherently nonlinear dynamics of water waves. It makes wave surface to be vertically asymmetric with flat troughs and sharp crests and, thus, also leads to similar overestimation. Numerous instrumental and retracking effects are usually related to tracker bias (TB). All the above effects are difficult to discriminate and describe within a consistent physical model which is relevant to the today altimetry needs.

Parametric models are widely used when SSB is represented as a function of geophysical predictors measured by altimeter itself or/and provided by sea wave models (e.g. WaveWatch III, see WAVEWATCH III Development Group, 2016). The parameterization in terms of the key predictor, significant wave height, $H_{s}$ provides rather good fit of the SSB (e.g. Bronner, Guillot and Picot, 2016, p.46) with the SSB coefficient $\alpha=S S B / H_{s}$ in the range of $2-$ 5\% (Gommenginger, Srokosz, Bellingham et al., 2018). Further corrections refer to the wind speed $U_{10}$ derived from the normalized radar cross-section $\sigma_{0}$ (NRCS) and the series in powers of $H_{s}$ and $U_{10}$ (e.g. Gaspar, Ogor, Le Traon et al., 1994). The parameterizations in terms of wind speed $U_{10}$ show, in particular, a pronounced regional dependence (Fu and Glazman, 1991) that can be explained mostly by the incompleteness of the set of predictors $H_{s}, U_{10}\left(\sigma_{0}\right)$ for wave dynamics and, then, for the SSB assessment. Using the characteristic wave period as an additional predictor shows some success in the empirical models of SSB (Pires, Fernandes, Gommenginger et al., 2016. 2018). However, the wave period, being a function of altimeter measured $H_{s}$ and $\sigma_{0}$ does not extend the number of independent physical predictors only changing the ansatz of the parametric formula.

The role of wave steepness (wave surface slope) as a physical predictor of sea state bias has been realized a long time ago (Longuet-Higgins, 1963 ; Srokosz, 1986; Glazman and Srokosz, 1991) and verified in case studies within wave forecasting models and in situ wave measurements (Millet, Arnold, Warnick et al., 2003; Gommenginger, Srokosz, Wolf et al., 2003b). The approach has not been applied to satellite data in the absence of wave steepness measurements by altimeters. A recent physical model of wave steepness from altimetry data (Badulin, 2014; Badulin, Grigorieva, Gavrikov et al., 2018) made this key parameter of wave dynamics readily available.

We present a physical analysis of SSB based on altimeter-derived wave steepness and pseudo-age. Wave steepness is defined in terms of $H_{s}$ and 
spectral peak period $T_{p}$ (cf. Gommenginger, Srokosz, Wolf et al., 2003b)

$$
\mu=\frac{\pi H_{s}}{g T_{p}^{2}} .
$$

and can be estimated from along-track derivatives of $H_{s}$ as follows (Badulin, 2014; Badulin, Grigorieva, Gavrikov et al., 2018)

$$
\mu=0.596\left|\nabla H_{s}\right|^{1 / 5} \text {. }
$$

In theoretical models of the EM scattering wave steepness is introduced as an integral (e.g. Gommenginger, Srokosz, Wolf et al., 2003b) through a range of contributing wave scales. However, the convergence of the integral requires physically relevant high-frequency cutoff. Definition of Eq. (1) is free of such problems but, the method of assessment of $\mu$ with Eq. (2) also puts certain limitations.

In contrast to the point-wise measured parameters $H_{s}$ and $\sigma_{0}$ wave steepness $\mu$ in Eq. (2) reflects a wave field evolution along the satellite track. On the one hand, Eq. (2) allows one to capture instantaneous sea state characteristics. On the other hand, the experimental estimates with Eq. (2) are vulnerable to higher random errors of the gradient $\nabla H_{s}$ as compared with its point-wise counterparts $H_{s}, \sigma_{0}$. As recompense, the gradient operation achieves error minimization of $H_{s}$ measurements and does not correlate with $H_{s}, \sigma_{0}$. Additionally, low exponent 1/5 in Eq. (2) reduces the random errors of the resulting estimates of $\mu$.

The simplest parametric model of SSB claims a proportionality to significant wave height

$$
S S B / H_{s}=\alpha_{l} \text {. }
$$

The coefficient $\alpha_{l}$ being typically in a range of $0.02-0.05(2-5 \%)$ (Gommenginger, Srokosz, Bellingham et al., 2018) provides a good reference but shows a dependence on chosen region and altimeter mission.

Advanced physical model of Fu and Glazman (1991) relates SSB with the so-called pseudo wave age

$$
\xi=\frac{g H_{s}}{U_{10}^{2}} .
$$

in a form of power-law dependence

$$
\frac{S S B}{H_{s}}=A\left(\frac{\xi}{\xi_{m}}\right)^{m}
$$


Parameters $A, m\left(\xi_{m}\right.$ - 'mean-over-globe' pseudo-age) in Eq. (5) also show a pronounced regional variations (see Table 1 in Fu and Glazman, 1991) and reflects insufficiency of the only altimeter-derived parameter to describe variability of SSB with required accuracy.

In $\S 2$ we propose a consistent similarity approach for wind-driven seas (e.g. Kitaigorodskii, 1962) for the specific problem of sea state bias in altimetry measurements. The approach is based on altimeter-derived dimensionless wave steepness $\mu$ and pseudo-age $\xi$. The use of dimensionless variables instead of dimensional $H_{s}, U_{10}$ implies a more transparent physical analysis of the combined effects on SSB.

$\S 3$ is focused on the analysis of satellite data. Data of the TOPEX/Poseidon follow-on missions Jason-1,2,3 (Ku-band $\lambda=2.2 \mathrm{~cm}$ ) and of SARAL/AltiKa Ka-band radar $(\lambda=0.8 \mathrm{~cm})$ shows good prospects of developing the parametric models based on $\xi, \mu$.

Discussion outlines the results and perspectives of the proposed approach.

\section{Similarity approach for the sea state bias in altimetry measure- ments}

\subsection{Dimensionless parameters as physical predictors of the sea state bias}

Assessment of sea state bias in altimetry is a complex multidisciplinary problem. Peculiarities of nonlinear water wave dynamics, electromagnetic scattering from the random sea surface, data assimilation and processing heavily affect the resulting ranging errors. Historically the SSB models rely upon altimeter-derived dimensional values of significant wave height $H_{s}$ and the normalized radar cross-section (NRCS) $\sigma_{0}$. Conversion of these measurable parameters into near-surface wind speed $U_{10}$ allows for associating results with in-situ sea state (e.g. Gommenginger, Srokosz, Challenor et al., 2003a; Quilfen, Chapron and Serre, 2004; Mackay, Retzler, Challenor et al., 2008). However, $U_{10}$ being estimated with parametric models contributes an additional error up to 20\% (Ardhuin, Stopa, Chapron et al., 2019).

A consistent physical approach can be developed based on the wellelaborated similarity approach. By following this approach the number of arguments of a physical problem can be reduced by a number of independent physical dimensions (the so-called П-theorem, see Barrenblatt, 1979). The non-dimensionalization appears to be a valuable research tool even (in our case, especially) if the form of the governing equations is still unknown. 
Potentially, the similarity approach can help to associate the physical effects with underlying physical mechanisms.

Existing models of SSB propose the following set of dimensional values:

1. $g$ - gravity acceleration responsible for occurrence sea surface waves;

2. $H_{s}$ - significant wave height directly measured by altimeter;

3. $U_{10}$ - altimeter-derived wind speed that depends mostly on dimensionless NRCS $\sigma_{0}$ measured by altimeter.

Two independent dimensions of the above set provides the only dimensionless parameter $\xi=g H_{s} / U_{10}^{2}$, the so-called pseudo-age of wind-driven waves. Based on physical variables $1-3$ the similarity approach gives a simple model of SSB (cf. Eq. (4) and Fu and Glazman, 1991)

$$
\frac{S S B}{H_{s}}=F_{1}(\xi)
$$

Eq. (6) can be generalized by introducing the altimeter-derived wave steepness $\mu$ (Badulin, 2014, Badulin, Grigorieva, Gavrikov et al., 2018)

$$
\frac{S S B}{H_{s}}=F_{2}(\xi, \mu)
$$

Pseudo-age $\xi$ and wave steepness $\mu$ are naturally associated with wind-wave coupling and inherently nonlinear wave dynamics but they cannot completely describe SSB. However, accounting additional physical effects inevitably complicate the model.

As an example, incorporating the well-known effect of radar wavelength $\lambda_{r}$ on the SSB (e.g. Melville, Stewart, Keller et al., 1991; Walsh, Jackson, Hines et al., 1991; Gommenginger, Srokosz, Wolf et al., 2003b) results in the extension of Eqs. (6:7)

$$
\frac{S S B}{H_{s}}=F_{3}(\xi, \mu, \nu)
$$

with the third dimensionless argument $\nu$ that can be presented in two different ways

$$
\nu_{1}=\frac{U_{10}^{2}}{g \lambda_{r}} ; \quad \nu_{2}=\frac{H_{s}}{\lambda_{r}} .
$$

The second form $\nu_{2}$ in Eq. (9) is preferable because it uses the directly measured wave height and not the empirically estimated value of wind speed 
like in $\nu_{1}$. Additionally, being dependent on square of wind speed $U_{10}$, the variable $\nu_{1}$ implies higher random errors than $\nu_{2}$.

The three-parametric dependencies Eq. (8) are justified when comparing measurements of radars with different wavelengths (e.g. Ku-band $\lambda \approx 2.2 \mathrm{~cm}$ vs Ka-band $\lambda \approx 0.8 \mathrm{~cm}$ ). However, in our case when the altimeter wavelength is fixed, the two-parametric dependency Eq. (7) is enough. Note, that conventional counterparts also operate with two-dimensional parameterizations and can be formally reduced to the following form

$$
S S B=F_{\text {conv }}\left(\nu_{1}, \nu_{2}\right) \text {. }
$$

Such a representation can be considered a special case of the formal application of the similarity approach, or an expression of a completely different physical conception, when the main role is given to the processes of electromagnetic scattering ( $\lambda_{r}$ and the associated electromagnetic bias EMB) while the role of wave dynamics (steepness $\mu$ and skewness bias SB) is belittled.

\subsection{Pseudo-age of wind-driven waves: stages of wave growth and SSB effects}

The first argument of $F_{2}$ in Eq. (7), pseudo-age $\xi$, is widely used in sea wave studies since Sverdrup and Munk (1947, see Fig.7 therein). Basic similarity theory of wind-driven waves (Kitaigorodskii, 1962) predicts its dependence on alternative dimensionless parameter, wave age $a=C_{p} / U_{10}=$ $g T_{p} /\left(2 \pi U_{10}\right)$ ( $T_{p}$ being wave spectra peak period and $C_{p}$ is the corresponding wave phase speed). This dependence is not universal and can heavily depend on features of wind-wave coupling: stratification of wind flow, wind gustiness etc. (see discussion in Abdalla and Cavaleri, 2002; Donelan, Babanin, Young et al., 2005: Badulin, Babanin, Resio et al., 2007).

At specific stages of wave field evolution, the dependencies $\xi(a)$ follow power laws quite well. Exponents and pre-exponents of these dependencies have found their consistent treatment within the weak turbulence theory (e.g. Zakharov, 2018, and refs. therein) supported by solid experimental background (Toba, 1972; Hasselmann, Ross, Müller et al., 1976; Zakharov and Zaslavsky, 1983a). Wave age $a$ as a ratio of wave and wind characteristic speeds remains a more effective parameter of physical analysis: $a \lesssim 1$ is treated as a case of growing wind seas while $a \gtrsim 1$ is referred to swell. A more detailed scheme of wave evolution can be developed based on the results of Gagnaire-Renou, Benoit and Badulin (2011); Badulin and Geogdzhaev (2019). 
In case of young seas $\left(a \ll 1\right.$, generally $a<1 / 3, \xi \sim a^{5 / 3}$, Hasselmann, Ross, Müller et al., 1976; Badulin and Geogdzhaev, 2019, see Fig.1 and Table 1) short and steep wind waves of relatively small amplitudes provide a minor contribution into SSB through a mechanism of SB (skewness bias). At the same time, high local wave steepness and the resulting white-capping can increase dramatically the effect of EMB (electromagnetic bias) both in terms of absolute and normalized value $S S B / H_{s}$.

Growing wind seas in the range of $1 / 3 \lesssim a \lesssim 1, \xi \sim a^{3 / 2}$ are associated with the so-called Toba regime (Toba, 1972). Increasing wave height amplifies absolute SB while wavelength growth (spectra downshift) partially suppresses this effect for normalized SSB.

At high ages (old waves $a>1$ ), wave growth and spectral downshifting is slowing down, then passing to hypothetical fully developed (matured) sea (Komen , Hasselmann and Hasselmann, 1984) or/and to swell. Skewness bias (SB), thus, is stabilizing or slowly decaying while the effect of EMB cannot be treated so straightforwardly. White-capping can be suppressed at this stage as well as sea surface drag coefficient (Kudryavtsev and Makin, 2011) and the effect of EMB can be reduced accordingly.

On the one hand, the dependence of SSB on pseudo-age $\xi$ (age $a$ ) shows rich physics. On the other hand, this physics implies a non-trivial coupling of EMB and SB fractions of SSB. The theoretical and experimental issues of discrimination of these fractions in the context of sea state bias are discussed below.

\subsection{Wave steepness: skewness bias of random weakly non-gaussian sea}

In contrast to multiple physical effects associated with pseudo-age $\xi$ wave steepness can be inherently related to the effect of skewness bias. Analysis of EM scattering from a weakly non-gaussian random surface by Glazman and Srokosz (1991) can be extended in view of parameterization Eq. (7).

The outcome of the analysis (Longuet-Higgins, 1963; Jackson, 1979; Srokosz, 1986) for satellite altimetry is expressed by the following relationship (see also Gommenginger, Srokosz, Wolf et al., 2003b)

$$
S S B=\frac{1}{8}\left(\frac{\lambda_{0}}{3}+\lambda_{1}\right) H_{s}
$$

in notations of Glazman and Srokosz (1991). Here $H_{s}=4\left\langle\zeta^{2}\right\rangle^{1 / 2}$ is defined in terms of sea surface elevation $\zeta, \lambda_{0}=\left\langle\zeta^{3}\right\rangle /\left\langle\zeta^{2}\right\rangle^{3 / 2}$ is the surface skewness, 
$\lambda_{1}$ was called 'cross-skewness' by Srokosz (1986)

$$
\lambda_{1}=\frac{\lambda_{120}+\lambda_{102}-2 \lambda_{011} \lambda_{111}}{1-\lambda_{011}^{2}} ; \lambda_{m n p}=\frac{\mu_{m n p}}{\mu_{200}^{m / 2} \mu_{020}^{n / 2} \mu_{002}^{p / 2}} ; \mu_{m n p}=\left\langle\zeta^{m} \zeta^{n} \zeta^{p}\right\rangle .
$$

and later referred as 'specular height' (Glazman and Srokosz, 1991). It can be shown (Longuet-Higgins, 1963; Barrick and Lipa, 1985; Srokosz, 1986) that both $\lambda_{0}$ and $\lambda_{1}$ may be expressed in terms of integrals of the two-dimensional wavenumber spectrum. This feature of Eq. (11) has been realized in estimates of SSB for simulated and observed sea wave spectra (Gommenginger, Srokosz, Wolf et al., 2003b) and developing a wind-speed based physical model of SSB by Glazman and Srokosz (1991).

In the latter case, explicit forms of spectra have been used that rely on classic solutions of the weak turbulence theory (Zakharov and Filonenko, 1966; Zakharov and Zaslavsky, 1983b a) and on empirical dependencies of wave energy on wave age $a=C_{p} / U_{10}$. For unidirectional sea the corresponding algebra leads to explicit analytical expressions for $\lambda_{0}, \lambda_{1}$ and power-law dependence of SSB on wave age (pseudo-age) in the form of Eq. (5) (see Glazman and Srokosz, 1991, sects.4-8). Quite similar algebra can be realized for wave spectra written in the form (see notations in Glazman and Srokosz, 1991)

$$
E(k)=\beta g^{2} k^{-5 / 2-n} k_{p}^{-1 / 2+n} \Phi\left(\frac{k}{k_{p}}\right) .
$$

At exponent $n=0$ Eq. (13) is consistent with the classic direct cascade solution by Zakharov and Filonenko (1966) with spectra tail asymptote $k^{-5 / 2}$ and a shape described by function $\Phi$ of dimensionless wavenumber $k / k_{p}\left(k_{p}\right.$ - spectral peak wavenumber). The spectrum magnitude $\beta$ in Eq. (13), evidently, is related to wave steepness $\mu$ in Eq. (1) as follows

$$
P \beta=\mu^{2} ; \quad P=\int_{0}^{+\infty} x^{-5 / 2-n} \Phi(x) d x
$$

The integral term $P$ can be easily calculated numerically or even analytically (cf. Glazman and Srokosz, 1991, Eqs. $(2.2,5.7,7.10)$ ) for a particular spectral shape function. For wind wave spectra the result of the integration depends weakly on stage of wave growth, the corresponding property is known as spectral shape invariance (Hasselmann , Barnett, Bouws et al., 1973; Hasselmann, Ross, Müller et al., 1976). Taking this property into account one 
can easily show that terms $\lambda_{0}, \lambda_{1}$ in Eq. (11) and the resulting SSB can be written in the form

$$
\lambda_{0} \sim A(\xi) \mu ; \quad \lambda_{1} \sim B(\xi) \mu ; \quad S S B \sim C(\xi) \mu H_{s}
$$

where wave steepness $\mu$ describes general linear dependence of the skewness bias (11) while functions of pseudo-age $A(\xi), B(\xi), C(\xi)$ represent the property of spectral shape invariance. The relatively weak variations of the coefficients of proportionality $A(\xi), B(\xi)$ in Eq. (13) and, hence, $C(\xi)$ in Eq. (11) with stage of wave development can be quantified using empirical dependencies of wave spectra parameters (shape function $\Phi$ ) on sea state (e.g. Babanin and Soloviev, 1998).

Eq. (15) provides a ground for the theoretical assessment of the skewness bias associated with the altimeter-derived wave steepness $\mu$. As far as wave steepness $\mu$ dramatically affects the wave breaking and, thus, the EMB, the experimental observation of the proportionality laws given by Eqs. (15) can be questionable (cf. Gommenginger, Srokosz, Wolf et al., 2003b).

\section{SSB data of Jason-3 and SARAL/AltiKa within dimensional and dimensionless formulations}

This section is related to carry out the SSB analysis with respect to two pairs of parameters: dimensional wave height and wind speed, and dimensionless pseudo-wave age $\xi$ and wave steepness $\mu$. Jason-1,2,3 and SARAL/Altika missions are taken into consideration as testing datasets. It should be stressed, that the SSB series of the SGDR Level 2 products cannot be considered as 'an ultimate truth'. The corresponding parametric models provide the best fit in space of dimensional $H_{s}$ and $U_{10}$ but, in general case, do not guarantee a reasonable result in space of dimensionless pair $\xi, \mu$. Moreover, wind speed $U_{10}$ is taken from empirical models and represents the most probable value in contrast to instantaneously measured wave height $H_{s}$ and its gradient $\nabla H_{s}$. Thus, dimensionless pair $\xi, \mu$ provides rather

physical than statistical method of SSB assessment (see discussion Badulin, Grigorieva, Gavrikov et al., 2018).

Firstly, we focus on specific properties of wave steepness $\mu$ (Eq. 2) as an argument of the SSB parametric model. The universality of its geographical and statistical distributions and weak dependence on the altimeter band (Jason vs SARAL/AltiKa missions) makes it a reliable and robust predictor for SSB. 
The next step of the analysis is finding out the similarity and robustness of SSB distributions themselves as functions of the newly proposed variables.

\subsection{Data description}

The data used here has been retrieved from Sensor Geophysical Data Records (SGDR, Level 2 product) with 1-Hz sampling from the AVISO FTP website (https://www.aviso.altimetry.fr). The analysis was conducted for the 2018 year: full cycles $70-105$ for Jason-3 and cycles $116-124$ for SARAL/AltiKa mission. The data provided the following parameters for further analysis:

1. Ka-band and Ku-band corrected (MLE3 retracking) significant wave height $H_{s}$;

2. sea state bias (SSB) correction in Ku- (Jason-3) and Ka-bands (SARAL/AltiKa);

3. altimeter wind speed;

4. coordinates (longitude, latitude) and time of standard 1-sec altimeter records.

Comparative analysis of the missions was mostly focused on the relevance of the dimensionless variables $\xi, \mu$ for the problem of SSB. Data of two pairs of missions Jason-1-Jason-2 (cycles 257 - 294 and 18 - 55, year 2009, correspondingly) and Jason-2-Jason-3 (cycles 18 - 55 for 2009 and 70 - 105, year 2018, correspondingly) were analyzed in order to exclude (or minimize) the effect of device dependence and to find out the similarity of the SSB distributions.

Following recommendations of Gavrikov, Krinitsky and Grigorieva (2016) the source track data were filtered. Along-track derivative of $H_{s}$ in Eq. (2) and the corresponding wave steepness $\mu$ was estimated as simple differences between two consecutive track points.

All data passed quality control procedure. After being filtered using the corresponding fields, e.g. ocean for variable surface_type, good for qual_alt_1hz_swh_ku_mle3 etc., noises were identified and excluded. Additionally, the homogeneity quality control check eliminated pseudo-age parameter over a 95 percentile. Hereafter, the computations were done for $0.5 \mathrm{~m}<H_{s}<8 \mathrm{~m},|S S B|<0.5 \mathrm{~m}, 1 \mathrm{~m} / \mathrm{s}<U_{10}<20 \mathrm{~m} / \mathrm{s}$. Only ice-free data for latitudes below $60^{\circ}(\mathrm{N}, \mathrm{S})$ were taken for the analysis.

Here we do not present analysis of the measurement errors of $H_{s}$ and accuracy of estimates of $U_{10}$ referring to generally accepted values $0.4 \mathrm{~m}$ 
and $1.5 \mathrm{~m} / \mathrm{s}$, correspondingly (e.g. Lavrova, Kostianoy, Lebedev et al., 2011; Dumont, Rosmorduc, Carrère et al., 2016b).

\subsection{General characteristics of altimeter-derived parameters}

As a preliminary step of the analysis, the normalized probability density functions (PDF) have been calculated for key measurable and derived parameters. Fig.1 shows results for the pair of missions Jason-3 - SARAL/AltiKa, year 2019 .

Significant wave heights of both missions show similar smooth distributions with a well-pronounced maximum of about $2 \mathrm{~m}$ (not shown here). Wind speed PDFs (not shown) demonstrate some discrepancies in shape, especially for $0.5 \mathrm{~m} / \mathrm{s}$ bin size, which is, however, less than the accuracy of wind speed in altimeter measurements (Lavrova, Kostianoy, Lebedev et al., 2011; Bonnefond, Haines and Watson, 2011). The most probable values of both missions remain close and fall within $6-8 \mathrm{~m} / \mathrm{s}$. Dimensionless pseudo-age $\xi$ (Fig.1 $1 a, b)$ also demonstrates an apparent agreement for both missions.

PDFs of wave steepness $\mu$ estimated by Eq. (2) (Fig, 1e,f) differ in the magnitude and peak positions but still look similar in shape. This similarity can be justified with a simple one-parametric transformation. Multiplication of the SARAL/AltiKa estimates of $\mu$ by a factor 1.159 makes both distributions identical in the whole range of $\mu$ with the perfectly matched peak positions (Fig, 1 $e, f$ ). Such a remarkable transformation can be explained by the geometry of satellite tracks (see discussion in Badulin, Grigorieva, Gavrikov et al., 2018). The near-pole orbit of SARAL/AltiKa (inclination $98.54^{\circ}$ ) makes estimates of $\mu$ more sensitive to predominantly zonal directions of wind and waves. Thus, SARAL/AltiKa tends to underestimate $\mu$ by approximately $15 \%$ of magnitudes as compared to Jason-3 with the orbit inclination $66^{\circ}$. The similarity of wave steepness PDFs of two missions is proved by global distributions of wave steepness in Fig,2. Being originally close in spatial patterns (Fig,2a) Jason-3 and Saral/AltiKa demonstrate an impressive quantitative agreement after re-scaling procedure in Fig $2 b$. Averaged over $4^{\circ} \times 4^{\circ}$ boxes $\mu$ varies in rather narrow range (Figs $2 a, b$ ), extreme values differ by less than $20 \%$ of maximal magnitudes for both missions. Recently, this general property has been treated as a universality of 'wave steepness climate' by Badulin, Grigorieva, Gavrikov et al. (2018).

Fig. $2 c$ presents normalized deviation of estimates of $\mu$ for Jason-3 $\left(\mu_{J 3}\right)$ 

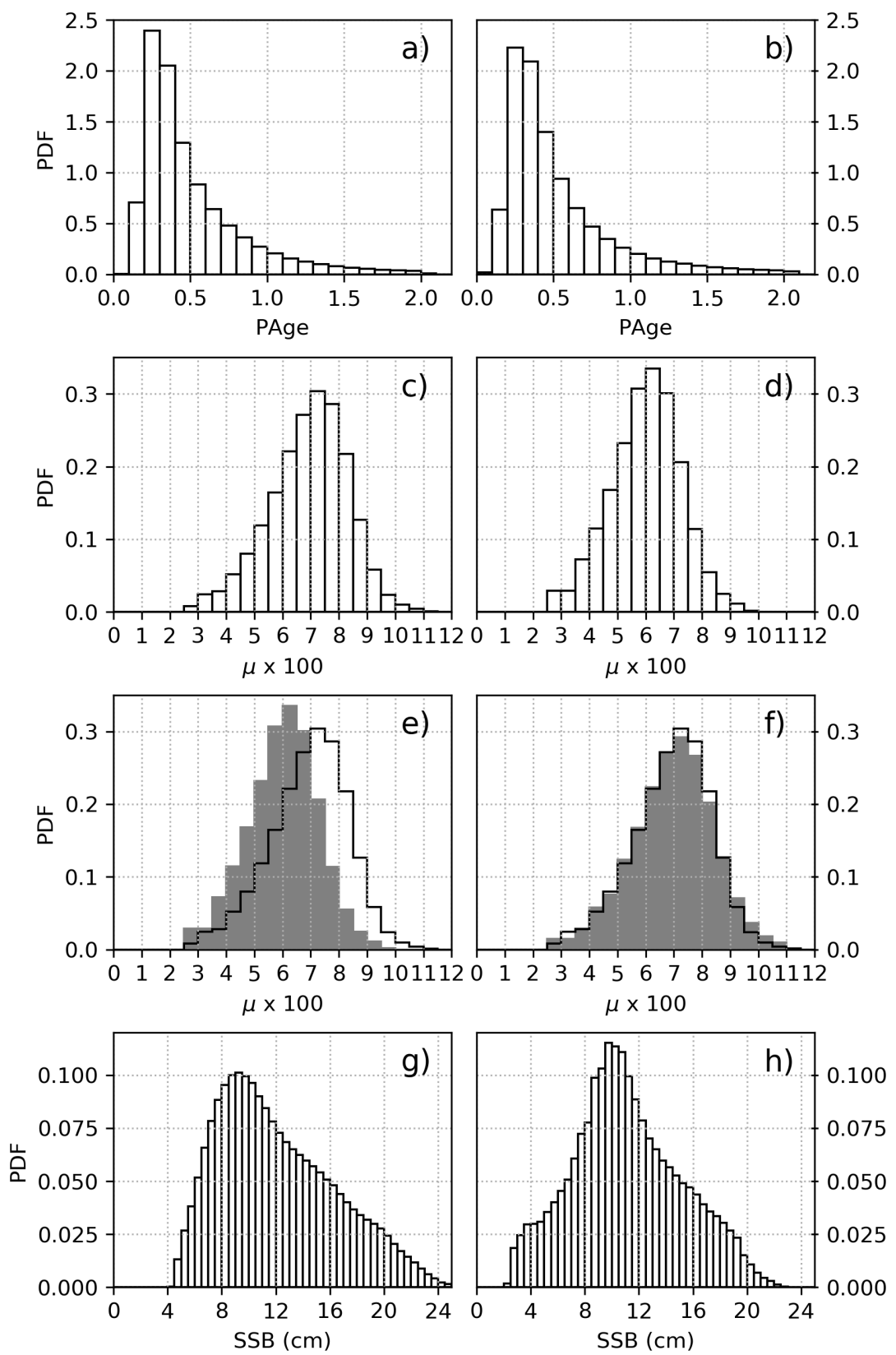

Figure 1: Probability density functions of altimeter-derived dimensionless values for Jason3 (left column) and SARAL/AltiKa (right column) for the year 2018. a,b) - pseudo-age $\left.\xi=g H_{s} / U_{10}^{2} ; \mathrm{c}, \mathrm{d}\right)$ - wave steepness $\left.\mu ; \mathrm{e}\right)$ - comparison of PDFs of two missions: unshaded curve - Jason-3, shaded - SARAL/AltiKa; $\mathrm{h}$ ) - the same as $\mathrm{h}$ ) with the re-scaled $\mu$ of SARAL/AltiKa $\left.\left(\mu_{\text {new }}=1.159 \mu\right) ; \mathrm{g}, \mathrm{h}\right)$ - normalized sea state bias $S S B / H_{s}$. 
and corrected SARAL/AltiKa $\left(\mu_{S A}\right)$. This deviation defined as

$$
\Delta \mu=100 \times \frac{\mu_{J 3}-\mu_{S A}}{\mu_{S A}}
$$

does not exceed $4 \%$ of local magnitudes in $95 \%$ of coordinate boxes in Fig. (1). Note, that the deviation is mostly positive in the Northern Hemisphere (wave steepness of Jason-3 is higher than one of SARAL/AltiKa) and negative in the Southern Hemisphere. Additional efforts are needed to make clear the origin of these differences.

The consistency of both missions in terms of PDFs of wave height $H_{s}$, wind speed $U_{10}$, pseudo-age $\xi$ and wave steepness $\mu$ appears in visible contrast with the SSB estimates (fig $19, h)$. Jason-3 with its long-time history of followon missions (since launching TOPEX/Poseidon in 1992) provides a smooth PDF. Oppositely, the relatively short record of the SARAL/AltiKa shows a rather sharp distribution with shifted probability maximum and spurious tails.

Similar statistical distributions for missions Jason-1,2 (not shown here) are in perfect agreement. In fact, this is because that follow-on missions of Jason instruments have the same thorough calibration and data retracking methods with close characteristics.

The above simple analysis of global distributions provides a ground for discussion of similarity and deficiency of SSB models in terms of conventional dimensional $\left(H_{s}, U_{10}\right)$ and dimensionless $(\xi, \mu)$ values.

\subsection{Conventional approach for SSB: dimensional arguments $H_{s}$ and $U_{10}$}

Efficiency of the SSB mapping as a function of dimensional arguments $H_{s}, U_{10}$ has shown its practical value (e.g. Pires, Fernandes, Gommenginger et al. 2018, and refs. therein) when comparing with non-parametric

crossover-based models. Fig $3 a, c, d$ illustrates a transparent consistency of $S S B\left(H_{s}, U_{10}\right)$ distribution for Jason-1,2,3 and one for TOPEX/Poseidon (cf. Vandemark, Tran, Beckley et al., 2002, Fig.1). Bin sizes $0.25 \mathrm{~m} / \mathrm{s}$ in $U_{10}$ and $0.25 \mathrm{~m}$ in $H_{s}$ were taken the same as in the cited paper. All the patterns demonstrate a gradual increase of $S S B$ with the increasing $H_{s}$ and wind speed. Local peculiarities at high values of wind speed and wave height in Vandemark, Tran, Beckley et al. (2002, Fig.1) are smoothed in the Jasons' patterns. The found correspondence of Jason-1,2,3 as follow-on missions of the TOPEX/Poseidon seems natural because of a long duration of missions and great amount of data underlying the estimates of SSB. 

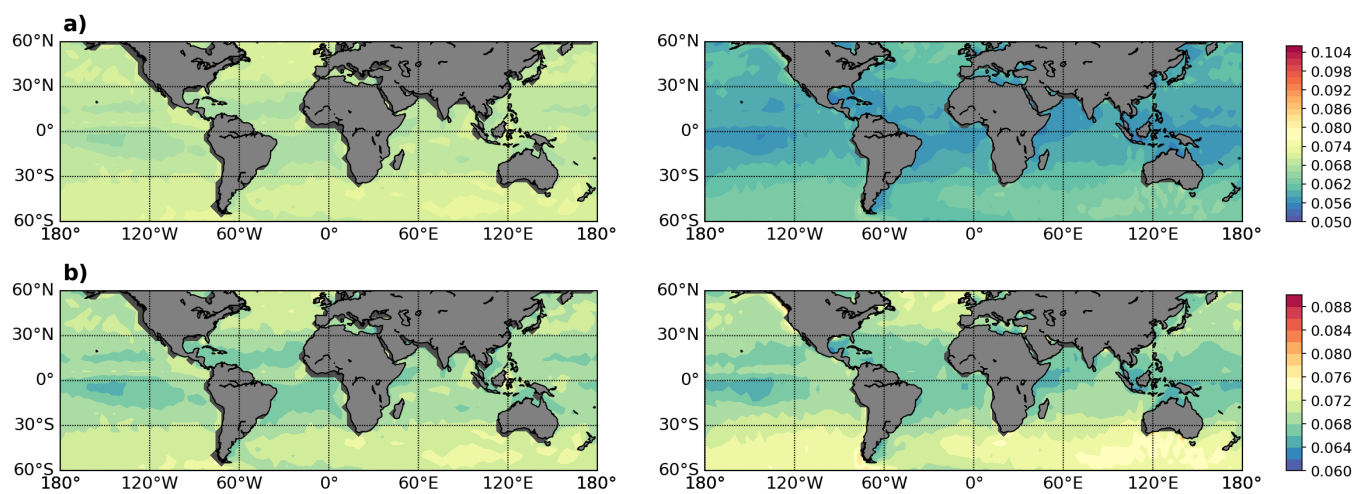

c)

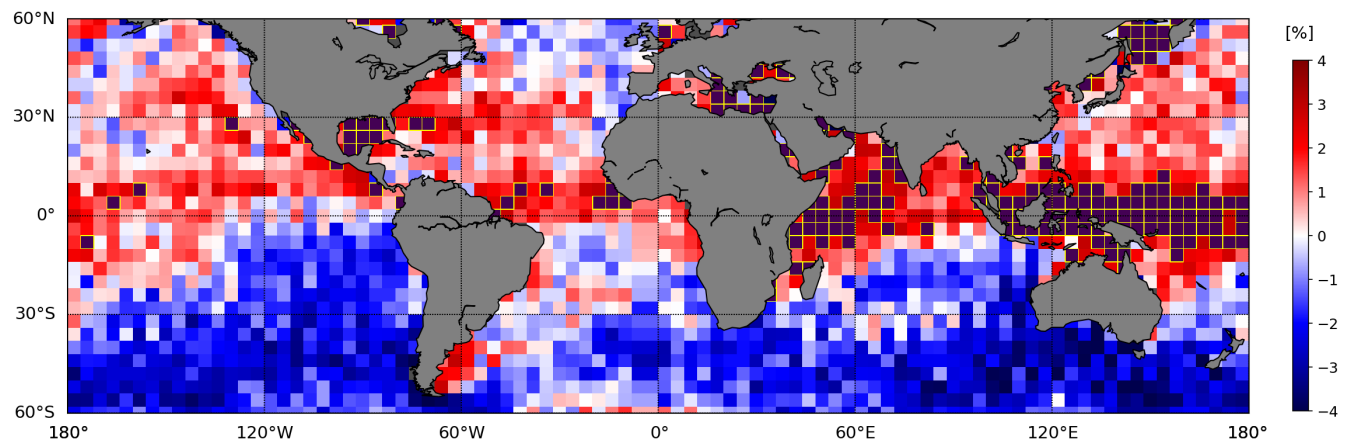

Figure 2: a) - global distribution of mean over $4^{\circ} \times 4^{\circ}$ coordinate boxes wave steepness $\mu$ Jason-3 (left column) and SARAL/AltiKa (top panels). b) - the same for SARAL/AltiKa wave steepness re-scaled by factor 1.159. c) - normalized difference of wave steepness of Jason-3 and re-scaled SARAL/AltiKa defined by Eq. 16. The outliers exceeding $4 \%$ are shown in violet. 

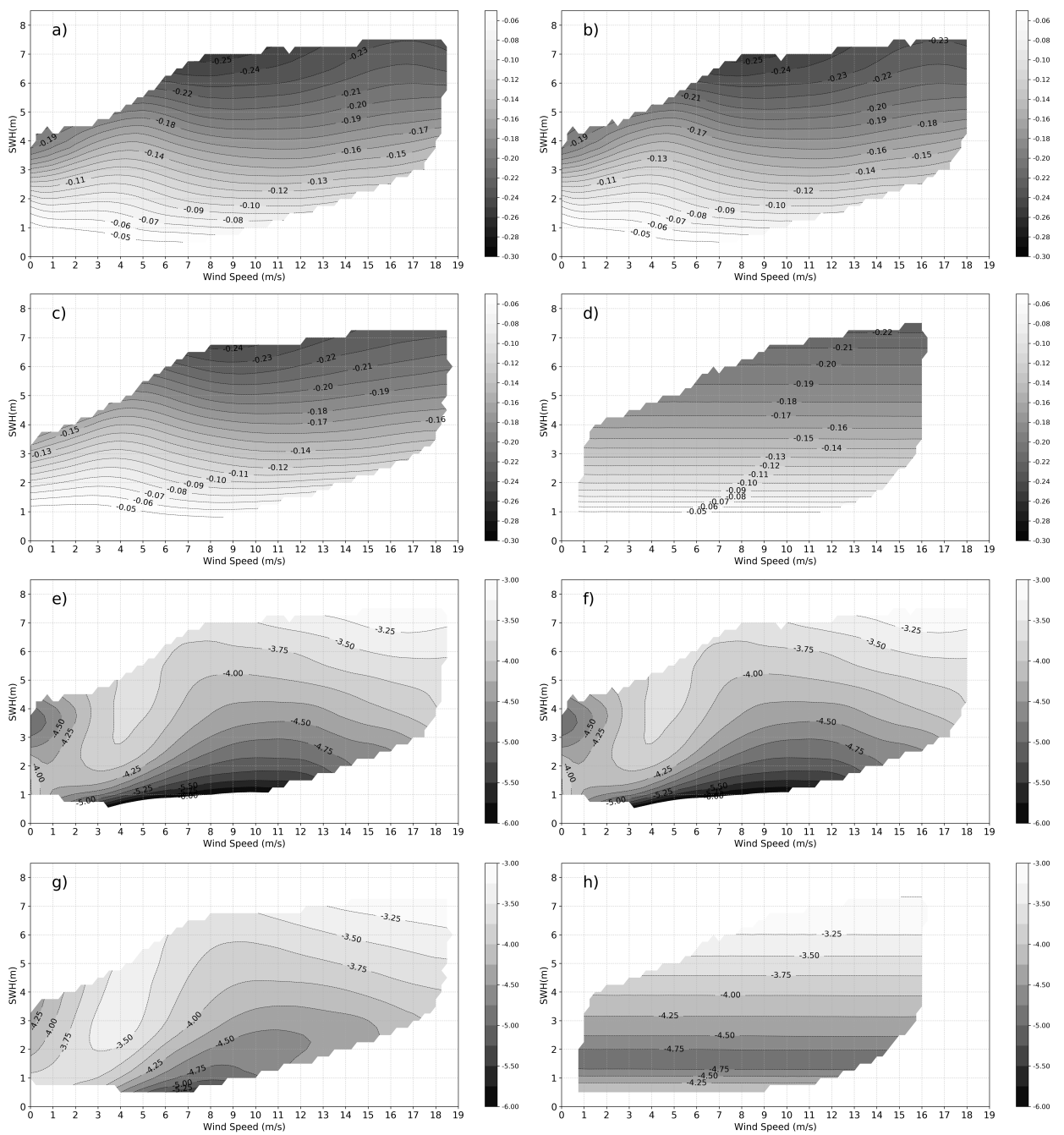

Figure 3: a,b,c,d) - isolines for the global $S S B$ (in meters) obtained from bin-averaging into boxes of width $(0.25 \mathrm{~m} / \mathrm{s}, 0.25 \mathrm{~m})$ over the $\left(H_{s}, U_{10}\right)$ domain. a) - Jason-3, cycles $70-105$, b) - Jason-2, cycles 18 - 55; c) - Jason-1, cycles 257-294; d) - SARAL/AltiKa, cycles 116 - 124 (cf. Vandemark, Tran, Beckley et al., 2002, Fig.1); e,f,g,h) - isolines for normalized value $S S B / H_{s}$ (in percents) over the $\left(H_{s}, U_{10}\right)$ domain for the same data series. 

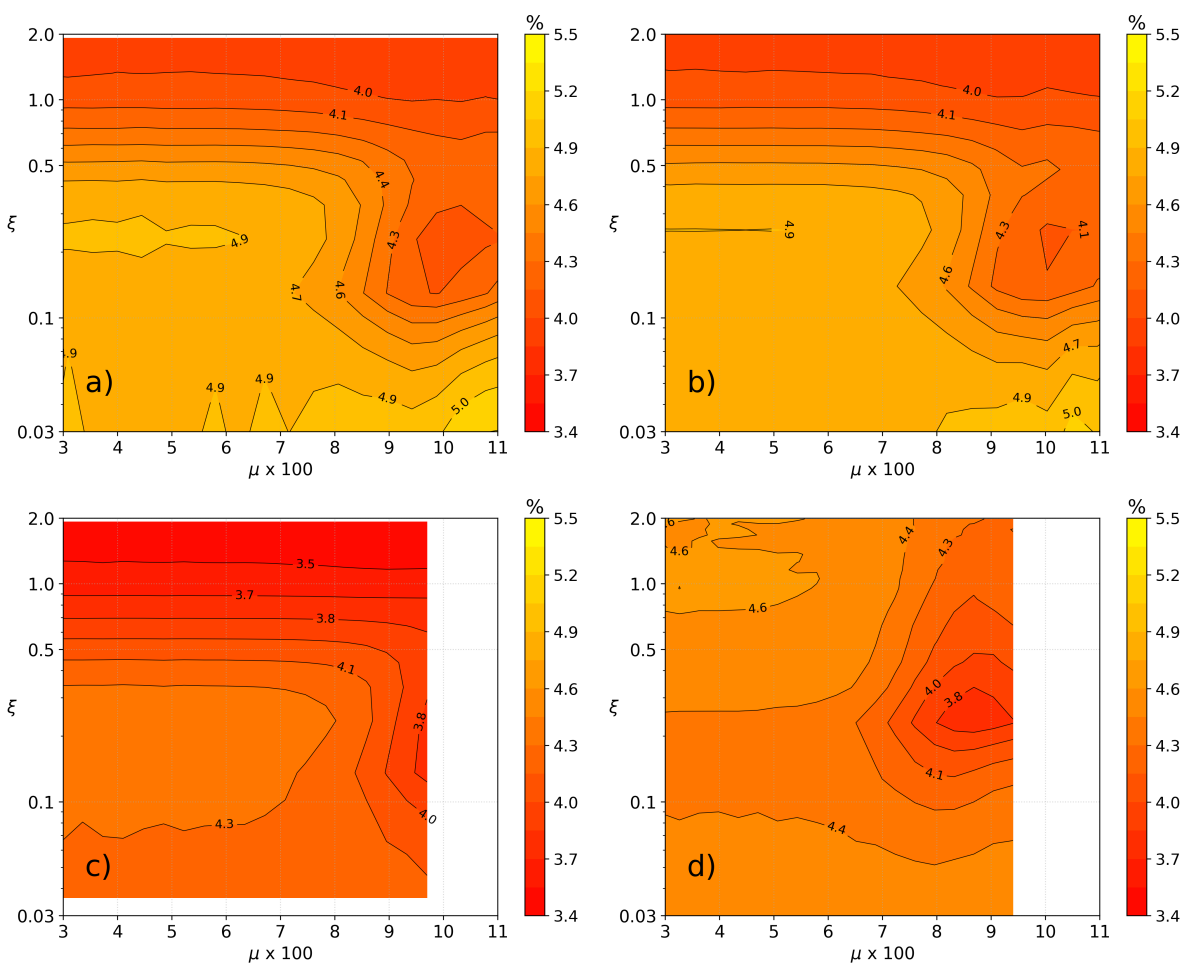

Figure 4: Isolines for the global normalized value $S S B / H_{s}$ (in percents) obtained from bin-averaging into boxes of width $\Delta \xi / \xi=1.1, \Delta \mu=0.002$. a) - Jason-3, cycles $70-105$, b) - Jason-2, cycles 18 - 55; c) - Jason-1, cycles 257 - 294; d) - SARAL/AltiKa, cycles $116-124$

Similar patterns for dimensionless $S S B / H_{s}$ are identical for Jason-2 and 3 (cf. Fig. 3e,h and sects. 5.6.3 in Dumont, Rosmorduc, Carrère et al., $2016 \mathrm{a}$ b). However, difference of the distribution of $S S B / H_{s}$ for Jason-1 caused by different retracking methods (MLE3 retracking for Jason-2,3) become more pronounced.

The SARAL/AltiKa mission (Fig $3 b, d$ ) with its relatively short duration of measurements portrays a different pattern when the SSB does not depend on wind speed $U_{10}$. Physical treatment of both Jason and SARAL/AltiKa distributions in terms of dimensional $H_{s}, U_{10}$ is quite simple: higher waves as well as stronger winds provide higher SSB. Collecting more data could improve the situation. 

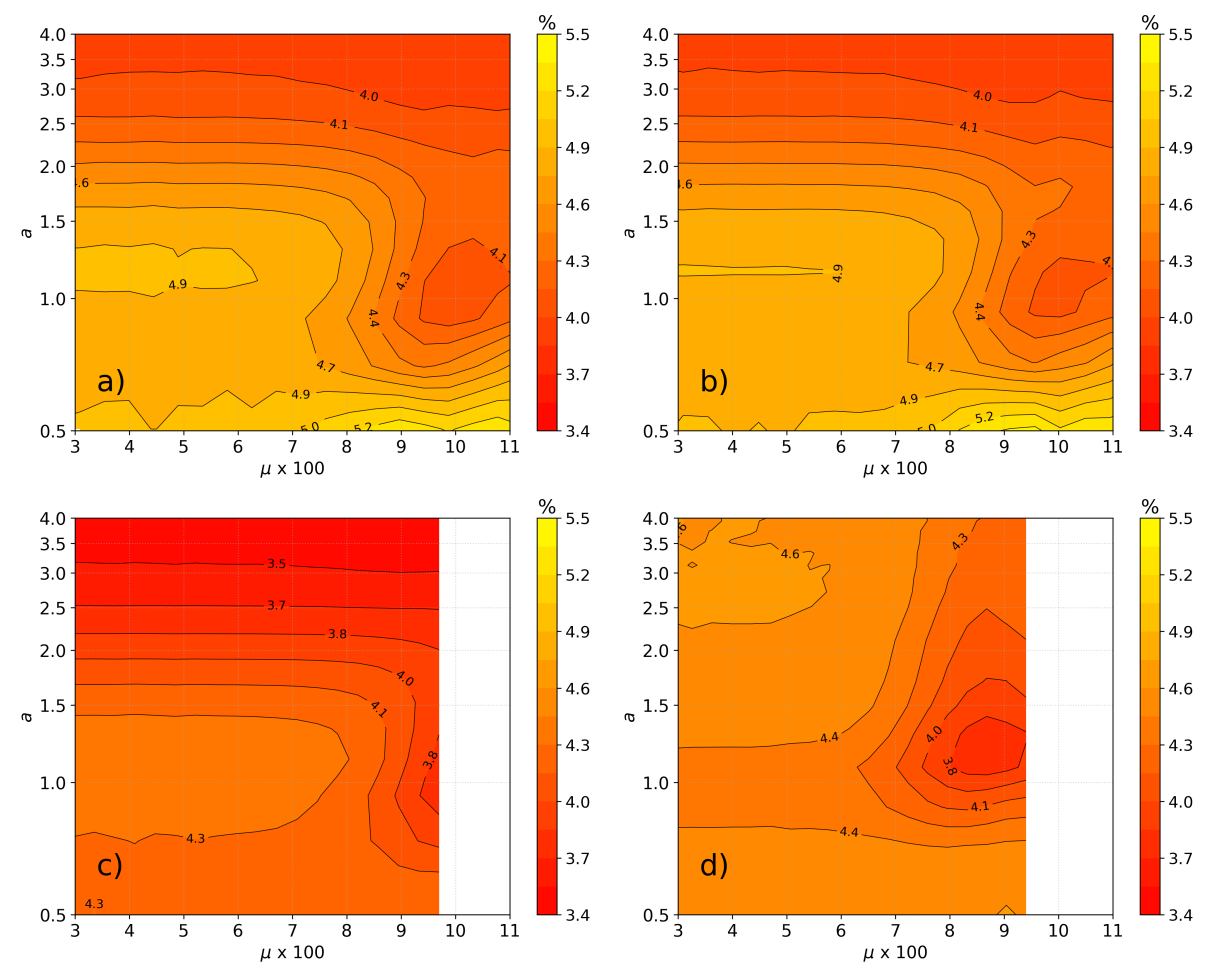

Figure 5: Same as previous for wave age $a=g T_{p} /\left(2 \pi U_{10}\right)$ 


\subsection{SSB within dimensionless $\xi, \mu$ : a step towards a physical analysis}

As it was mentioned above, re-casting SSB onto $(\xi, \mu)$ does not guarantee the best data fit in this new argument space. But the analysis of resulting SSB distributions for various data sets provides the basis for further development of the proposed approach.

Fig, 4 presents bin-averaged normalized values $S S B / H_{s}$. Fig, 4 for Jasons and SARAL/AltiKa (Fig.4 year 2018, $\mu$ is corrected by factor 1.159, see sect. 3.2) show different patterns as expected for altimeters operating in different bands. In turns, all the Ku-band Jason's missions in Fig 4 $4 a, b, c$ (Jason-1, Jason-2, year 2009 and Jason-3, year 2018) show definite similarity in shapes of SSB patterns (palettes are different in Fig. 4). Moreover, Jason-2 and Jason-3 demonstrate perfect agreement both in shapes and magnitudes. Different years (2009 and 2018) make such an agreement to be a strong argument for our approach: various data samples show robustness of distributions of $\operatorname{SSB}(\xi, \mu)$.

The partial success with re-mapping all the Jason's missions $(1-3)$ can be referred to the effect of re-tracking (TB - tracking bias): MLE3 retracking data has been applied for Jason-2,3 missions only.

Similar maps for the pair $a, \mu$ (wave age - wave steepness) of all four missions are presented in Fig.5. While the wave age $a$ is not measured directly an empirical parameterization $a(\xi)$ has been used following Kahma and Calkoen (1994) (see also Zakharov, Badulin, Geogjaev et al., 2019). This re-mapping allows for relating SSB with stages of wave growth described in sect. 2.2. All the missions show extrema in classic transition from wind sea to swell at

$a \approx 1$. At $\mu \gtrsim 0.07$ and wave ages $a \gtrsim 0.7$ the dimensionless $S S B / H_{s}$ shows a breakup for all missions. This domain of $(\xi, \mu)$-plane corresponds to the tongue' of normalized SSB in Fig $3 e, f, g$ at relatively low wind speed $U_{10}$ and, hence, might indicate issues in wind measurements by altimeters.

The dispersion of normalized SSB (not shown) is large enough and, in some cases, even comparable with the range of variation of the $S S B / H_{s}$ itself. The low confidence level accumulates not only the random errors of $\xi$ and $\mu$ but the uncertainties of $\left(H_{s}, U_{10}\right)$-based parametric models. Complete retracking of SSB in terms of the new arguments $\xi, \mu$ would be helpful to resolve this issue.

All Figs. 45 do not contain any domains of linear dependence of the normalized value $S S B / H_{s}$ on wave steepness (see Eq. 15). In fact, the model

of sect. 2.3 describes just a partial effect of wave dynamics on SSB: skewness 
bias (SB) due to Stokes-like asymmetry of water wave profiles. The analysis shows that this effect is probably compensated by other effects that also dependent on wave steepness, first of all, by electromagnetic bias (EMB).

\section{Conclusions and Discussion}

The following list of conclusions and remarks overviews the results of the paper:

1. The basic idea of this work was to explore a physical relevance of unconventional parameterization of sea state bias (SSB). Two dimensionless arguments, wave pseudo-age $\xi$ defined by Eq. (3) and the wave slope $\mu$ (Eq.4) derived from along-track derivatives, were introduced within the consistent similarity analysis. The new approach has not evolved into alternative model of SSB so far. Such a project requires a complete retracking a wealth of altimeter data in terms of $\xi$ and $\mu$ instead of the conventional dimensional parameters $H_{s}$ and $U_{10}$.

2. One comparative advantage is shown with similarity of wave steepness distributions for Jason missions and SARAL/AltiKa operating at different bands. It should be emphasized that $\mu$ captures instantaneous sea state in contrast to wind speed $U_{10}$ based on the parametric models providing the most probable values.

3. A potential prospects of new approach were examined on readily available SSB records of recent altimetry missions Jason-1,2,3 and SARAL/AltiKa. The conventional parametric $\left(H_{s}, U_{10}\right)$-based models are not obliged to generate any consistent patterns in $(\xi, \mu)$-space. Nevertheless, our analysis shows quite consistent patterns in the dimensionless coordinates and provide an opportunity for their physical explanation.

4. The similarity of SSB patterns in $(\xi, \mu)$-space for missions Jason-2 and Jason-3 for different data sets (years 2009 and 2018) proved robustness of the corresponding dependencies. At the same time, their dissimilarity with mission Jason-1 pointed out the problem of instrumentation and calibration (retracking) effects on the resulting SSB.

5. The theoretical consideration of sect.2 found the expected linear dependence of SSB on $\mu$ : skewness bias (SB) of a random wave field is proportional to wave steepness like in the case of the classic stationary Stokes wave. Previous attempts to quantify such dependence as a contribution of one of the constituents of SSB, the skewness bias (SB), have been 
successful (Gommenginger, Srokosz, Wolf et al., 2003b). Nevertheless, our theoretical result has not been supported by the data analysis. The most likely explanation is that all the effects contributing to SSB (e.g. EMB, SB, TB) are closely interrelated and hardly discriminated within the parametric models.

\section{Acknowledgements}

Open access data of the portal AVISO (http://www.aviso.altimetry.fr/en/home.html) has been used in this work. The data analysis (sect. 3) has been supported by the Russian Foundation for Basic Research $\sharp 19-05-00147$. Theoretical study of sect. 2 has been carried out under the Russian Science Foundation grant 'Turbulence and coherent structures in the integrable and nonintegrable systems’ $\sharp 19-72-30028$ and Migo Group (http://migogroup.ru). The authors are thankful to reviewers of the manuscript for their helpful critics.

\section{References}

\section{References}

Abdalla, S., Cavaleri, L., 2002. Effects of wind variability and variable air density on wave modeling. J. Geophys. Res. 107, C7, 3080. doi:10.1029/ 2000JC000639.

Ardhuin, F., Stopa, J.E., Chapron, B., Collard, F., Husson, R., Jensen, R.E., Johannessen, J., Mouche, A., Passaro, M., Quartly, G.D., Swail, V., Young, I., 2019. Observing sea states. Frontiers in Marine Science 6, 124. doi:10.3389/fmars.2019.00124.

Babanin, A.V., Soloviev, Y.P., 1998. Field investigation of transformation of the wind wave frequency spectrum with fetch and the stage of development. J. Phys. Oceanogr. 28, 563-576.

Badulin, S., Grigorieva, V., Gavrikov, A., Geogjaev, V., Krinitskiy, M., Markina, M., 2018. Wave steepness from satellite altimetry for wave dynamics and climate studies. Russ. J. Earth. Sci. 18, ES5005. doi:10.2205/ 2018ES000638.

Badulin, S.I., 2014. A physical model of sea wave period from altimeter data. J. Geophys. Res. Oceans 119, 856-869. doi:10.1002/2013JC009336. 
Badulin, S.I., Babanin, A.V., Resio, D., Zakharov, V., 2007. Weakly turbulent laws of wind-wave growth. J. Fluid Mech. 591, 339-378.

Badulin, S.I., Geogdzhaev, V.V., 2019. Evaluation of wind wave growth parameters basing on spectral fluxes. Radiophysics and Quantum Electronics 61, 545-552. doi:10.1007/s11141-019-09915-8.

Barrenblatt, G.I., 1979. Scaling, self-similarity, and intermediate asymptotics: Dimensional analysis and intermediate asymptotics. Plenum Press, New York/London.

Barrick, D., Lipa, B., 1985. Analysis and interpretation of altimeter sea echo. Advances in Geophysics 27, 60-100. doi:10.1016/S0065-2687(08) 60403-3.

Bonnefond, P., Haines, B., Watson, C., 2011. In situ absolute calibration and validation: a link from coastal to open-ocean altimetry, in: Vignudelli, S., Kostianoy, A., Cipollini, P., Benveniste, J. (Eds.), Coastal Altimetry. Springer, Berlin, Heidelberg. 259-296. doi:10.1007/978-3-642-12796-0.

Bronner, E., Guillot, A., Picot, N., 2016. SARAL/AltiKa Products Handbook. Technical Report 2 rev. 5. CNES, ISRO.

Donelan, M.A., Babanin, A.V., Young, I.R., Banner, M.L., McCormick, C., 2005. Wave follower field measurements of the wind input spectral function. Part I. Measurements and calibrations. J. Atmos. Ocean. Technol. 22, 799813.

Dumont, J.P., Rosmorduc, V., Carrère, L., Picot, N., Bronner, E., Couhert, A., Desai, S., Bonekamp, H., Scharroo, R., Lillibridge, J., Leuliette, E., 2016a. OSTM/Jason-2 Products Handbook. Technical Report 1, rev 10. CNES : SALP-MU-M-OP-15815-CN, EUMETSAT : EUM/OPSJAS/MAN/08/0041, JPL : OSTM-29-1237, NOAA/NESDIS : Polar Series/OSTM J400.

Dumont, J.P., Rosmorduc, V., Carrere, L., Picot, N., Bronner, E., Couhert, A., Guillot, A., Desai, S., Bonekamp, H., Figa, J., Scharroo, R., Lillibridge, J., 2016b. Jason-3 Products Handbook. Technical Report 1 rev.2. NASA, JPL, CNES, NOAA, EUMETSAT. 
Fu, L.L., Glazman, R., 1991. The effect of the degree of wave development on the sea state bias in radar altimetry measurement. J. Geophys. Res. 96, 829-834.

Gagnaire-Renou, E., Benoit, M., Badulin, S.I., 2011. On weakly turbulent scaling of wind sea in simulations of fetch-limited growth. J. Fluid Mech. $669,178-213$.

Gaspar, P., Ogor, F., Le Traon, P.Y., Zanife, O.Z., 1994. Estimating the sea state bias of the Topex and Poseidon altimeters from crossover differences. J. Geophys. Res. 99, 24,981-24,994.

Gavrikov, A.V., Krinitsky, M.A., Grigorieva, V.G., 2016. Modification of satellite altimetry database Globwave for diagnosis of sea wave fields. Oceanology 56, 301-306.

Glazman, R.E., Srokosz, M.A., 1991. Equilibrium wave spectrum and sea state bias in satellite altimetry. J. Phys. Oceanogr. 21, 1609-1621.

Gommenginger, C., Srokosz, M., Bellingham, C., Snaith, H., Pires, N., Fernandes, M.J., Tran, N., Vandemark, D., Moreau, T., Labroue, S., Scharroo, R., 2018. Sea state bias: 25 years on, Presentation at and Abstract in: 25 years of progress in radar altimetry, 24-29 September 2018, Ponta Delgada, São Miguel Island Azores Archipelago, Portugal.

Gommenginger, C.P., Srokosz, M.A., Challenor, P.G., Cotton, P.D., 2003a. Measuring ocean wave period with satellite altimeters: A simple empirical model. Geophys. Res. Lett. 30, 2150. doi:10.1029/2003GL017743.

Gommenginger, C.P., Srokosz, M.A., Wolf, J., Janssen, P.A.E.M., 2003b. An investigation of altimeter sea state bias theories. J. Geophys. Res. 108, 3011. doi:10.1029/2001JC001174.

Hasselmann, K., Barnett, T.P., Bouws, E., Carlson, H., Cartwright, D.E., Enke, K., Ewing, J.A., Gienapp, H., Hasselmann, D.E., Kruseman, P., Meerburg, A., Muller, P., Olbers, D.J., Richter, K., Sell, W., Walden, H., 1973. Measurements of wind-wave growth and swell decay during the Joint North Sea Wave Project (JONSWAP). Dtsch. Hydrogh. Zeitschr. Suppl. 12 . 
Hasselmann, K., Ross, D.B., Müller, P., Sell, W., 1976. A parametric wave prediction model. J. Phys. Oceanogr. 6, 200-228.

Jackson, F.C., 1979. The reflection of impulses from a nonlinear random sea. J. Geophys. Res. 84, 4939-4943. doi:10.1029/JC084iC08p04939.

Kahma, K.K., Calkoen, C.J., 1994. Growth curve observations, in: Komen, G.J., Cavaleri, L., Donelan, M., Hasselmann, K., Hasselmann, S., Janssen, P. (Eds.), Dynamics and modeling of ocean waves. Cambridge University Press, Cambridge, New York, Melbourne, Madrid, Cape Town, Singapore, Saõ Paulo, pp. 74-182.

Kitaigorodskii, S.A., 1962. Applications of the theory of similarity to the analysis of wind-generated wave motion as a stochastic process. Bull. Acad. Sci. USSR, Geophys. Ser., Engl. Transl. N1, 105-117.

Komen, G.J., Hasselmann, S., Hasselmann, K., 1984. On the existence of a fully developed wind-sea spectrum. J. Phys. Oceanogr. 14, 1271-1285.

Kudryavtsev, V.N., Makin, V.K., 2011. Impact of ocean spray on the dynamics of the marine atmospheric boundary layer. Boundary-Layer Meteorol. 140, 383-410. doi:10.1007/s10546-011-9624-2.

Lavrova, O.Y., Kostianoy, A.G., Lebedev, S.A., Mityagina, V.I., Ginzburg, A.I., Sheremet, N.A., 2011. Complex satellite monitoring of the Russian seas. Space Research Institute of the Russian Academy of Sciences, Moscow. ISBN: 978-5-9903101-1-7. In Russian.

Longuet-Higgins, M.S., 1963. The effect of non-linearities on statistical distributions in the theory of sea waves. J. Fluid Mech. 17, 459-480.

Mackay, E.B.L., Retzler, C.H., Challenor, P.G., Gommenginger, C.P., 2008. A parametric model for ocean wave period from $\mathrm{Ku}$-band altimeter data. J. Geophys. Res. 113, C03029. doi:10.1029/2007JC004438.

Melville, W. K., I. R. H. Stewart, Keller, W. C., Kong, J. A., Arnold, D. V., I. A. T. Jessupi, Loewen, M. E., Slinn, A., 1991. Measurements of electromagnetic bias in radar altimetry. J. Geophys. Res. 96, C3, 4915-4924.

Millet, F.W., Arnold, D.V., Warnick, K.F., Smith, J., 2003. Electromagnetic bias estimation using in situ and satellite data: 1. RMS wave slope. J. Geophys. Res. 108, 3040. doi:10.1029/2001JC001095. 
Pires, N., Fernandes, M.J., Gommenginger, C., Scharroo, R., 2016. A conceptually simple modeling approach for Jason-1 sea state bias correction based on 3 parameters exclusively derived from altimetric information. Remote Sens. 8, 1-13. doi:10.3390/rs8070576.

Pires, N., Fernandes, M.J., Gommenginger, C., Scharroo, R., 2018. Improved sea state bias estimation for altimeter reference missions with altimeteronly three-parameter models. IEEE Trans. on Geosci. and Remote Sensing, 57, 1448-1462, doi:10.1109/TGRS.2018.2866773.

Quilfen, Y., Chapron, B., Serre, M., 2004. Calibration/validation of an altimeter wave period model and application to TOPEX/Poseidon and Jason-1 altimeters. Marine Geodesy 27, 535-549.

Srokosz, M.A., 1986. On the joint distribution of surface elevation and slopes for a nonlinear random sea, with an application to radar altimetry. J. Geophys. Res. 91, 995-1006.

Sverdrup, H.V., Munk, W.H., 1947. Wind, sea, and swell: Theory of relations for forecasting. Hydrographic Office Pub. 60, U.S. Navy.

Toba, Y., 1972. Local balance in the air-sea boundary processes. Part I. On the growth process of wind waves. J. Oceanogr. Soc. Japan 28, 109-121.

The WAVEWATCH III Development Group (WW3DG). 2016 User manual and system documentation of WAVEWATCH III version 5.16. Tech. Note 329, NOAA/NWS/NCEP/MMAB. College Park, MD, USA, 326 pp. + Appendices.

Vandemark, D., Tran, N., Beckley, B.D., Chapron, B., Gaspar, P., 2002. Direct estimation of sea state impacts on radar altimeter sea level measurements. J. Geophys. Res. 29, 2148. doi:10.1029/2002GL015776.

Walsh, E. J., Jackson, F. C., Hines, D. E., Piazza, C., Hevizi, L. G., McLaughlin, D. J., McIntosh, R. E., Swift, R. N., Scott, J. F., Yungel, J. K., Frederick, E. B., 1991. Frequency dependence of electromagnetic bias in radar altimeter sea surface range measurements. Journal of Geophysical Research: Oceans 96 (C11), 20571-20583. doi:10.1029/91JC02097.

Zakharov, V., 2018. Analytic theory of a wind-driven sea. Procedia IUTAM $26,43-58$. 
Zakharov, V.E., Badulin, S.I., 2011. On energy balance in wind-driven seas. Doklady Earth Sciences 440, 1440-1444.

Zakharov, V.E., Badulin, S.I., Geogjaev, V.V., Pushkarev, A.N., 2019. Weakturbulent theory of wind-driven sea. Earth and Space Science 6. doi:10. 1029/2018EA000471.

Zakharov, V.E., Filonenko, N.N., 1966. Energy spectrum for stochastic oscillations of the surface of a fluid. Soviet Phys. Dokl. 170, 1292-1295.

Zakharov, V.E., Zaslavsky, M.M., 1983a. Dependence of wave parameters on the wind velocity, duration of its action and fetch in the weak-turbulence theory of water waves. Izv. Atmos. Ocean. Phys. 19, 300-306.

Zakharov, V.E., Zaslavsky, M.M., 1983b. Shape of spectrum of energy carrying components of a water surface in the weak-turbulence theory of wind waves. Izv. Atmos. Ocean. Phys. 19, 207-212. 